\title{
New existence theory of positive solutions to fourth order $p$-Laplacian elasticity problems
}

\author{
Yaling Zhou and XueMei Zhang*
}

$\overline{{ }^{*} \text { Correspondence: zxm74@sina.com }}$ Department of Mathematics and Physics, North China Electric Power University, Beijing, 102206, People's Republic of China

\begin{abstract}
Using a new technique for dealing with the bending term of the fourth order $p$-Laplacian elasticity problems, several new and more general results are obtained for the existence of at least single, twin or triple positive solutions. It is interesting to point out that estimates on the norms of these solutions will also be provided.
\end{abstract}

Keywords: fourth order $p$-Laplacian elasticity problems; transformation technique; positive solutions; estimate on the norm

\section{Introduction}

It is well known that many nonlinear problems can be reduced to the search for solutions of various differential equations. Fixed point methods are often used for proving the existence of such solutions. An overview of such results can be found in Guo et al. [1], in Guo and Lakshmikantham [2], and in Demling [3].

At the same time, we notice that some new techniques via appropriate transformation are proved to be very effective in studying the solvability of differential equations. Such techniques have attracted the attention of Zhang et al. [4] and Wong [5], etc. In [4], Zhang et al. considered the existence of positive solutions of the following problems:

$$
\left\{\begin{array}{l}
x^{(n)}(t)+f\left(t, x(t), x^{\prime}(t), \ldots, x^{(n-2)}(t)\right)=\theta, \quad t \in J, t \neq t_{k}, k=1,2, \ldots, m \\
\left.\Delta x^{(n-1)}\right|_{t=t_{k}}=-I_{k}\left(x^{(n-2)}\left(t_{k}\right)\right), \quad k=1,2, \ldots, m, \\
x^{(i)}(0)=\theta, \quad i=0,1, \ldots, n-3 \\
x^{(n-2)}(0)=x^{(n-2)}(1)=\int_{0}^{1} g(t) x^{(n-2)}(t) d t
\end{array}\right.
$$

where $J=[0,1]$.

First, by means of the linear transformation

$$
x^{(n-2)}(t)=y(t)
$$

the authors converted (1.1) into

$$
\left\{\begin{array}{l}
x^{(n-2)}(t)=y(t), \quad t \in J, \\
x^{(i)}(0)=\theta, \quad i=1,2, \ldots, n-3,
\end{array}\right.
$$

(c) 2015 Zhou and Zhang. This article is distributed under the terms of the Creative Commons Attribution 4.0 International License (http://creativecommons.org/licenses/by/4.0/), which permits unrestricted use, distribution, and reproduction in any medium, provided you give appropriate credit to the original author(s) and the source, provide a link to the Creative Commons license, and indicate if changes were made. 
and

$$
\left\{\begin{array}{l}
-y^{\prime \prime}(t)=f\left(t, x(t), x^{\prime}(t), \ldots, x^{(n-2)}(t)\right), \quad t \in J, t \neq t_{k} \\
\left.\Delta y^{\prime}\right|_{t=t_{k}}=-I_{k}\left(y\left(t_{k}\right)\right), \quad k=1,2, \ldots, m \\
y(0)=y(1)=\int_{0}^{1} g(t) y(t) d t .
\end{array}\right.
$$

Then it follows from Lemma 2.3 in [4] that they converted the results obtained for problem (1.2) to the counterpart for problem (1.2).

In [5], Wong transformed the following problems:

$$
\left\{\begin{array}{l}
(-1)^{m} y^{(2 m+1)}(t)=F\left(t, y(t), y^{\prime}(t)\right), \quad 0<t<1, \\
y(0)=0, \quad y^{(2 k-1)}(0)=y^{(2 k-1)}(1)=0, \quad 0 \leq k \leq m
\end{array}\right.
$$

into

$$
\left\{\begin{array}{l}
y^{\prime}(t)=x(t), \quad t \in J \\
y(0)=0
\end{array}\right.
$$

and

$$
\left\{\begin{array}{l}
(-1)^{m} x^{(2 m)}(t)=F\left(t, y(t), y^{\prime}(t)\right), \quad 0<t<1 \\
x^{(2 k-2)}(0)=x^{(2 k-2)}(1)=0, \quad 1 \leq k \leq m
\end{array}\right.
$$

by using

$$
y^{\prime}(t)=x(t)
$$

So the existence of a solution of the complementary Lidstone boundary value problem (1.3) follows from the existence of a solution of the Lidstone boundary value problem (1.4). From the proof of the main results of [5], we notice that the above paper requires $F$ to satisfy some assumptions of monotonicity which are essential for the technique used.

Being directly inspired by $[4,5]$, in the present paper, by using a transformation technique and a fixed point method, we shall investigate the existence and multiplicity of positive solutions for the fourth order $p$-Laplacian elasticity problems

$$
\left\{\begin{array}{l}
\left(\phi_{p}\left(y^{\prime \prime}(t)\right)\right)^{\prime \prime}=F\left(t, y(t), y^{\prime \prime}(t)\right), \quad 0<t<1, \\
a y(0)-b y^{\prime}(0)=\int_{0}^{1} g(s) y(s) d s, \\
a y(1)+b y^{\prime}(1)=\int_{0}^{1} g(s) y(s) d s, \\
\phi_{p}\left(y^{\prime \prime}(0)\right)=\phi_{p}\left(y^{\prime \prime}(1)\right)=\int_{0}^{1} h(s) \phi_{p}\left(y^{\prime \prime}(s)\right) d s,
\end{array}\right.
$$

where $a, b>0, \phi_{p}(t)=|t|^{p-2} t, p>1, \phi_{q}=\phi_{p}^{-1}, \frac{1}{p}+\frac{1}{q}=1, F:[0,1] \times R \times R \rightarrow R$ is continuous.

Problem (1.5) occurs in beam theory; see [6,7], for example for a beam with small deformation; a beam of a material which satisfies a nonlinear power-like stress and strain law; a beam with two-sided links which satisfies a nonlinear power-like elasticity law. If $p=2$, $g=0$ and $h=0$, in mechanics, problem (1.5) is called the cantilever beam equation $[8,9]$. The equation describes the deflection of an elastic beam fixed at the right and freed at the left. 
Most research papers on fourth order elasticity problems consider nonlinear terms that as regards $F$ in (1.5) involve $y$ only, and derivative-dependent nonlinearities are seldom tackled; see [10-18] to name a few. Besides achieving new results, the presence of the derivative $y^{\prime \prime}$ in the nonlinear term $F$ requires a special technique to tackle the problem.

The main features of this paper are as follows. First, compared with [6-18], besides achieving new results, estimates on the norms of these solutions will also be provided. Second, we transform problem (1.5) into a differential systems without bending term, i.e., the technique to deal with bending term is completely different from that of $[7,19-21]$. Finally, it is pointed out that we do not need any monotone assumption on $F$, which is weaker than the corresponding assumptions on $F$ in [5].

The rest of the paper is organized as follows: In Section 2, we first present some definitions and lemmas which are needed throughout this paper and then state several fixed point results: Krasnosel'skii's fixed point theorem in a cone, a new fixed point theorem due to Avery and Henderson and Leggett-Williams' fixed point theorem. In Section 3, we use Krasnosel'skii's fixed point theorem to obtain the existence of at least one or two positive solutions of problem (1.5). Section 4 will further discuss the existence of twin positive solutions of problem (1.5). Two new results will be presented by new fixed point theorem due to Avery and Henderson. Section 5 is due to develop existence criteria for (at least) three positive solutions of problem (1.5). In particular, our results in these sections are new when $p=2$ (the semilinear case). Finally, in Section 6, an example is included to verify the theoretical results. To the best of the authors' knowledge, it is the first paper where the transformation technique and fixed point method are applied to the fourth order $p$ Laplacian elasticity problems.

\section{Preliminaries}

To establish the existence of positive solutions of problem (1.5), let us list the following assumptions, which will hold throughout this paper:

$\left(\mathrm{H}_{1}\right) F \in C([0,1] \times[0,+\infty) \times(-\infty, 0],[0,+\infty))$;

$\left(\mathrm{H}_{2}\right) g, h \in L^{1}[0,1]$ are nonnegative, $\mu \in[0, a), v \in[0,1)$, where

$$
\mu=\int_{0}^{1} g(s) d s, \quad v=\int_{0}^{1} h(s) d s .
$$

We shall reduce elasticity problem (1.5) to a system without the bending term. To this goal, first, by means of the transformation

$$
\phi_{p}\left(y^{\prime \prime}(t)\right)=-x(t) .
$$

It follows from (1.5) that Lemma 2.1 holds.

Lemma 2.1 (See [22]) Assume that $\left(\mathrm{H}_{2}\right)$ holds. Then for any $x \in C[0,1]$, the boundary value problem

$$
\left\{\begin{array}{l}
-y^{\prime \prime}(t)=\phi_{q}(x(t)), \quad 0<t<1, \\
a y(0)-b y^{\prime}(0)=\int_{0}^{1} g(s) y(s) d s, \\
a y(1)+b y^{\prime}(1)=\int_{0}^{1} g(s) y(s) d s,
\end{array}\right.
$$


has a unique solution y given by

$$
y(t)=\int_{0}^{1} H_{1}(t, s) \phi_{q}(x(s)) d s
$$

where

$$
\begin{aligned}
& H_{1}(t, s)=G_{1}(t, s)+\frac{1}{a-\mu} \int_{0}^{1} G_{1}(s, v) g(v) d v, \\
& G_{1}(t, s)=\frac{1}{d} \begin{cases}(b+a s)(b+a(1-t)), & 0 \leq s \leq t \leq 1, \\
(b+a t)(b+a(1-s)), & 0 \leq t \leq s \leq 1,\end{cases} \\
& d=a(a+2 b) .
\end{aligned}
$$

Taking into account (2.1) and (2.2), problem (1.5) reduces to the following problem:

$$
\left\{\begin{array}{l}
x^{\prime \prime}(t)=-F\left(t, \int_{0}^{1} H_{1}(t, s) \phi_{q}(x(s)) d s,-\phi_{q}(x(t))\right), \quad 0<t<1, \\
x(0)=x(1)=\int_{0}^{1} h(s) x(s) d s .
\end{array}\right.
$$

Lemma 2.2 (See [22]) Assume that $\left(\mathrm{H}_{1}\right)-\left(\mathrm{H}_{2}\right)$ hold. Then the boundary value problem (2.5) has a unique solution $x$ given by

$$
x(t)=\int_{0}^{1} H_{2}(t, s) F\left(s, \int_{0}^{1} H_{1}(s, \tau) \phi_{q}(x(\tau)) d \tau,-\phi_{q}(x(s))\right) d s,
$$

where

$$
\begin{aligned}
& H_{2}(t, s)=G_{2}(t, s)+\frac{1}{1-v} \int_{0}^{1} G_{2}(s, v) h(v) d v \\
& G_{2}(t, s)= \begin{cases}s(1-t), & 0 \leq s \leq t \leq 1 \\
t(1-s), & 0 \leq t \leq s \leq 1 .\end{cases}
\end{aligned}
$$

If problem (2.5) has a solution $x^{*}$, then by (2.2), problem (1.5) has a solution given by

$$
y^{*}(t)=\int_{0}^{1} H_{1}(t, s) \phi_{q}\left(x^{*}(s)\right) d s
$$

So the existence of a solution of problem (1.5) follows from the existence of a solution of problem (2.5).

Lemma 2.3 (See [22]) Let $\left(\mathrm{H}_{2}\right)$ hold. Then we have the following results:

$$
\begin{aligned}
& G_{1}(t, s)>0, \quad H_{1}(t, s)>0, \quad \forall t, s \in[0,1] \\
& \frac{b^{2}}{d} \leq G_{1}(t, s) \leq G_{1}(s, s) \leq \frac{(a+b)^{2}}{d}, \quad \forall t, s \in[0,1] \\
& \rho_{1} \leq H_{1}(t, s) \leq H_{1}(s, s) \leq \rho_{2}, \quad \forall t, s \in[0,1]
\end{aligned}
$$


where

$$
\rho_{1}=\frac{b^{2} \gamma_{1}}{a+2 b}, \quad \rho_{2}=\frac{\gamma_{1}(a+b)^{2}}{a+2 b}, \quad \gamma_{1}=\frac{1}{a-\mu} .
$$

Lemma 2.4 (See [22]) Let $\left(\mathrm{H}_{2}\right)$ hold. Then we obtain the following results:

$$
\begin{aligned}
& G_{2}(t, s) \geq 0, \quad H_{2}(t, s) \geq 0, \quad \forall t, s \in[0,1], \\
& G_{2}(t, s)>0, \quad H_{2}(t, s)>0, \quad \forall t, s \in(0,1), \\
& G_{2}(t, t) G_{2}(s, s) \leq G_{2}(t, s) \leq G_{2}(s, s) \leq \frac{1}{4}, \quad \forall t, s \in[0,1], \\
& \rho G_{2}(s, s) \leq H_{2}(t, s) \leq \gamma_{2} G_{2}(s, s) \leq \frac{1}{4} \gamma_{2}, \quad \forall t, s \in[0,1],
\end{aligned}
$$

where

$$
\rho=\frac{\int_{0}^{1} G_{2}(\tau, \tau) h(\tau) d \tau}{1-v}, \quad \gamma_{2}=\frac{1}{1-v} .
$$

It is clear from (2.2) that $\left\|y^{*}\right\| \leq \rho_{2} \phi_{q}\left(\left\|x^{*}\right\|\right)$, moreover, if $x^{*}$ is positive, so is $y^{*}$.

Let $J=[0,1], E=C[0,1]$. It is well known that $E$ is a real Banach space with the norm $\|\cdot\|$ defined by $\|x\|=\max _{t \in J}|x(t)|$.

Define an operator $T: E \rightarrow E$ as follows:

$$
(T x)(t)=\int_{0}^{1} H_{2}(t, s) F\left(s, \int_{0}^{1} H_{1}(s, \tau) \phi_{q}(x(\tau)) d \tau,-\phi_{q}(x(s))\right) d s
$$

Let $K$ be a cone in $E$ which is defined as

$$
K=\{x \in E: x(t) \geq 0, \text { and } x(t) \geq \delta\|x\| \text { for } t \in J\}
$$

where

$$
\delta=\frac{\rho}{\gamma_{2}} .
$$

Lemma 2.5 Let $\left(\mathrm{H}_{1}\right)-\left(\mathrm{H}_{2}\right)$ hold. Then we have $T(K) \subset K$, and $T: K \rightarrow K$ is completely continuous.

Proof In view of condition (2.15), we see that

$$
\begin{aligned}
\|T x\| & =\max _{t \in J} \int_{0}^{1} H_{2}(t, s) F\left(s, \int_{0}^{1} H_{1}(s, \tau) \phi_{q}(x(\tau)) d \tau,-\phi_{q}(x(s))\right) d s \\
& \leq \int_{0}^{1} \gamma_{2} G_{2}(s, s) F\left(s, \int_{0}^{1} H_{1}(s, \tau) \phi_{q}(x(\tau)) d \tau,-\phi_{q}(x(s))\right) d s .
\end{aligned}
$$

Moreover, it follows from (2.15) that 


$$
\begin{aligned}
(T x)(t) & =\int_{0}^{1} H_{2}(t, s) F\left(s, \int_{0}^{1} H_{1}(s, \tau) \phi_{q}(x(\tau)) d \tau,-\phi_{q}(x(s))\right) d s \\
& \geq \int_{0}^{1} \rho G_{2}(s, s) F\left(s, \int_{0}^{1} H_{1}(s, \tau) \phi_{q}(x(\tau)) d \tau,-\phi_{q}(x(s))\right) d s \\
& \geq \delta\|T x\| .
\end{aligned}
$$

This proves that $T(K) \subset K$.

Next by standard methods and the Ascoli-Arzela theorem one can prove that $T: K \rightarrow K$ is completely continuous. So this is omitted.

In the rest of this section, we state several fixed point theorems which we needed later.

Lemma 2.6 (See [1-3]) Let $P$ be a cone of real Banach space E, and $\Omega_{1}$ and $\Omega_{2}$ be two bounded open sets in $E$ such that $0 \in \Omega_{1}$ and $\bar{\Omega}_{1} \subset \Omega_{2}$. Let operator $A: P \cap\left(\bar{\Omega}_{2} \backslash \Omega_{1}\right) \rightarrow P$ be completely continuous. Suppose that one of the two conditions

(i) $\|A x\| \leq\|x\|, \forall x \in P \cap \partial \Omega_{1}$ and $\|A x\| \geq\|x\|, \forall x \in P \cap \partial \Omega_{2}$,

(ii) $\|A x\| \geq\|x\|, \forall x \in P \cap \partial \Omega_{1}$ and $\|A x\| \leq\|x\|, \forall x \in P \cap \partial \Omega_{2}$ is satisfied. Then $A$ has at least one fixed point in $P \cap\left(\bar{\Omega}_{2} \backslash \Omega_{1}\right)$.

Remark 2.1 To make clear what $\bar{\Omega}_{2}, \partial \Omega_{2}, \partial \Omega_{1}$, and $\Omega_{2} \backslash \bar{\Omega}_{1}$ mean, we give typical examples of $\Omega_{1}$ and $\Omega_{2}, e . g$,

$$
\Omega_{1}=\left\{x \in C[a, b]:\|x\|_{\infty}<r\right\}, \quad \Omega_{2}=\left\{x \in C[a, b]:\|x\|_{\infty}<R\right\}
$$

with $0<r<R$, where $\|x\|_{\infty}=\sup _{t \in J}|x(t)|$.

Given a nonnegative continuous functional $\psi$ on a cone $P$ of a real Banach space, we define, for each $r>0$, the set

$$
P(\psi, r)=\{u \in P: \psi(u)<r\}
$$

Lemma 2.7 (See [23]) Let $P$ be a cone in a real Banach space. Let $\alpha$ and $\gamma$ be increasing nonnegative continuous functional on $P$, and let $\theta$ be a nonnegative continuous functional on $P$ with $\theta(0)=0$ such that, for some $c>0$ and $H>0$,

$$
\gamma(x) \leq \theta(x) \leq \alpha(x) \quad \text { and } \quad\|x\| \leq H \gamma(x)
$$

for all $x \in \overline{P(\gamma, c)}$. Suppose there exists a completely continuous operator $A: \overline{P(\gamma, c)} \rightarrow P$ and $0<r_{1}<r_{2}<$ c such that

$$
\theta(\lambda x) \leq \lambda \theta(x) \quad \text { for } 0 \leq \lambda \leq 1 \text { and } x \in \partial P\left(\theta, r_{2}\right)
$$

and

(i) $\gamma(A x)>$ c for all $x \in \partial P(\gamma, c)$;

(ii) $\theta(A x)<r_{2}$ for all $x \in \partial P\left(\theta, r_{2}\right)$;

(iii) $P\left(\alpha, r_{1}\right) \neq \emptyset$ and $\alpha(A x)>r_{1}$ for $x \in \partial P\left(\alpha, r_{1}\right)$. 
Then $A$ has at least two positive solutions $x_{1}$ and $x_{2}$ belonging to $\overline{P(\gamma, c)}$ satisfying

$$
r_{1}<\alpha\left(x_{1}\right) \text {, with } \theta\left(x_{1}\right)<r_{2} \text {, and } r_{2}<\theta\left(x_{2}\right) \text { with } \gamma\left(x_{2}\right)<c \text {. }
$$

Let $0<r_{1}<r_{2}$ be given and let $\beta$ be a nonnegative continuous concave functional on the cone $K$. Define the convex sets $K_{r_{1}}, K\left(\beta, r_{1}, r_{2}\right)$ by

$$
\begin{aligned}
& K_{r_{1}}=\left\{x \in K:\|x\|<r_{1}\right\}, \\
& K\left(\beta, r_{1}, r_{2}\right)=\left\{x \in K: r_{1} \leq \beta(x),\|x\| \leq r_{2}\right\} .
\end{aligned}
$$

Finally we state the Leggett-Williams fixed point theorem [24].

Lemma 2.8 (See [24]) Let K be a cone in a real Banach space $E, A: \bar{K}_{c} \rightarrow \bar{K}_{c}$ be completely continuous and $\beta$ be a nonnegative continuous concave functional on $K$ with $\beta(x) \leq\|x\|$ for all $x \in K_{r_{1}}$. Suppose there exist $0<d<r_{1}<r_{2} \leq c$ such that

(i) $\left\{x \in K\left(\beta, r_{1}, r_{2}\right): \beta(x)>r_{1}\right\} \neq \emptyset$ and $\beta(A x)>r_{1}$ for $x \in K\left(\beta, r_{1}, r_{2}\right)$;

(ii) $\|A x\|<d$ for $\|x\| \leq d$;

(iii) $\beta(A x)>r_{1}$ for $x \in K\left(\beta, r_{1}, c\right)$ with $\|A x\|>r_{2}$.

Then $A$ has at least three positive solutions $x_{1}, x_{2}, x_{3}$ satisfying

$$
\left\|x_{1}\right\|<d, \quad r_{1}<\beta\left(x_{2}\right), \quad\left\|x_{3}\right\|>d \quad \text { and } \quad \beta\left(x_{3}\right)<r_{1} .
$$

\section{Single or twin solutions of problem (1.5)}

In this section, we apply Lemma 2.6 to establish the existence of positive solutions for problem (1.5). We begin by introducing the following conditions on $F(t, u, v)$ :

$\left(\mathrm{H}_{3}\right)$ There exist two positive constants $r, R$ with $\left(\rho_{2}+1\right) \phi_{q}(r)<\left(\rho_{1}+1\right) \phi_{q}(\delta R)$ such that

$$
\begin{aligned}
& F(t, u, v) \leq \frac{1}{\eta} r, \quad \forall t \in J,|u|+|v| \leq\left(\rho_{2}+1\right) \phi_{q}(r) \\
& F(t, u, v) \geq \frac{1}{\rho \beta \delta} R, \quad \forall t \in J,|u|+|v| \geq\left(\rho_{1}+1\right) \phi_{q}(\delta R)
\end{aligned}
$$

where

$$
\eta=\frac{1}{4} \gamma_{2}, \quad \sigma=\rho_{1} \delta^{q-1}, \quad \beta=\int_{0}^{1} s(1-s) d s=\frac{1}{6} .
$$

Theorem 3.1 Assume that $\left(\mathrm{H}_{1}\right)-\left(\mathrm{H}_{3}\right)$ hold. Then we have the following conclusions:

(i) Problem (2.5) has (at least) one positive solution $x \in K$ such that

$$
\delta r \leq x(t) \leq \frac{1}{\delta} R, \quad t \in J .
$$

(ii) Problem (1.5) has (at least) one positive solution y such that

$$
\left\{\begin{array}{l}
y(t)=\int_{0}^{1} H_{1}(t, s) \phi_{q}(x(s)) d s, \quad t \in J \\
\|y\| \leq \rho_{2} \phi_{q}(\|x\|) ; \\
y(t) \geq \sigma \phi_{q}(\|x\|), \quad t \in J
\end{array}\right.
$$


We further have

$$
\sigma \phi_{q}(r) \leq y(t) \leq \rho_{2} \phi_{q}\left(\frac{1}{\delta} R\right), \quad t \in J
$$

Proof Let $T$ be the cone preserving, completely continuous operator that was defined by (2.16).

Let $x \in K$ with $\|x\|=r$. Then $0 \leq \phi_{q}(x(t)) \leq \phi_{q}(r), t \in J$, and

$$
0 \leq \int_{0}^{1} H_{1}(s, \tau) \phi_{q}(x(\tau)) d \tau \leq \rho_{2} \phi_{q}(r)
$$

And hence for $x \in K$ with $\|x\|=r$, we have

$$
\left|\int_{0}^{1} H_{1}(s, \tau) \phi_{q}(x(\tau)) d \tau\right|+\left|-\phi_{q}(x(t))\right| \leq\left(\rho_{2}+1\right) \phi_{q}(r)
$$

Then it follows from (3.1) that

$$
\begin{aligned}
\|T x\| & =\max _{t \in J} \int_{0}^{1} H_{2}(t, s) F\left(s, \int_{0}^{1} H_{1}(s, \tau) \phi_{q}(x(\tau)) d \tau,-\phi_{q}(x(s))\right) d s \\
& \leq \eta \frac{1}{\eta} r=r .
\end{aligned}
$$

Now if we let $\Omega_{1}=\{x \in K:\|x\|<r\}$, then (3.6) shows that

$$
\|T x\| \leq\|x\|, \quad x \in \partial \Omega_{1} .
$$

Further, let

$$
R_{1}=\frac{1}{\delta} R,
$$

and

$$
\Omega_{2}=\left\{x \in K:\|x\|<R_{1}\right\}
$$

Then $x \in K$ and $\|x\|=R_{1}$ implies

$$
x(t) \geq \delta x(s), \quad t, s \in J,
$$

that is,

$$
x(t) \geq \delta R_{1}=R, \quad t \in J .
$$

Hence, $\phi_{q}(x(t)) \geq \phi_{q}(R)$ for all $t \in J$, and

$$
\int_{0}^{1} H_{1}(s, \tau) \phi_{q}(x(\tau)) d \tau \geq \rho_{1} \int_{0}^{1} \phi_{q}(x(\tau)) d \tau \geq \rho_{1} \phi_{q}(\delta\|x\|) .
$$


So

$$
\left|\int_{0}^{1} H_{1}(s, \tau) \phi_{q}(x(\tau)) d \tau\right|+\left|-\phi_{q}(x(t))\right| \geq\left(\rho_{1}+1\right) \phi_{q}(\delta R) .
$$

Using condition (3.2), it follows from $x \in K$ and $\|x\|=R_{1}$ that

$$
\begin{aligned}
\|T x\| & =\max _{t \in J} \int_{0}^{1} H_{2}(t, s) F\left(s, \int_{0}^{1} H_{1}(s, \tau) \phi_{q}(x(\tau)) d \tau,-\phi_{q}(x(s))\right) d s \\
& \geq \rho \beta \frac{1}{\rho \beta \delta} R=\frac{R}{\delta}=R_{1},
\end{aligned}
$$

that is, $x \in \partial \Omega_{2}$ implies

$$
\|T x\| \geq\|x\|
$$

It follows from Lemma 2.6 that problem (2.5) has (at least) one positive solution $x \in$ $\bar{\Omega}_{2} \backslash \Omega_{1}$ satisfying (3.3).

It is observed from (2.2) that problem (1.5) has (at least) one positive solution $y$ such that

$$
y=\int_{0}^{1} H_{1}(t, s) \phi_{q}(x(s)) d s, \quad t \in J \text { and }\|y\| \leq \rho_{2} \phi_{q}(\|x\|) .
$$

Moreover, since $x \in K$, we get for $t \in J$,

$$
\begin{aligned}
y(t) & =\int_{0}^{1} H_{1}(t, s) \phi_{q}(x(s)) d s \\
& \geq \rho_{1} \int_{0}^{1} \phi_{q}(x(s)) d s \\
& \geq \rho_{1} \phi_{q}(\delta\|x\|) \\
& =\sigma \phi_{q}(\|x\|) .
\end{aligned}
$$

Then we get (3.4).

Further, it follows from (3.3) and (3.4) that (3.5) holds.

In Theorem 3.2 we assume the following condition on $F(t, u, v)$ :

$\left(\mathrm{H}_{4}\right)$ There exist two positive constants $r, R$ with $\left(\rho_{2}+1\right) \phi_{q}(r)<R$ such that:

$$
\begin{aligned}
& F(t, u, v) \geq \frac{1}{\rho_{1} \phi_{p}\left(\rho_{1}+1\right) \beta \delta} \phi_{p}(|u|+|v|), \quad \forall t \in J,|u|+|v| \leq\left(\rho_{2}+1\right) \phi_{q}(r) \\
& F(t, u, v) \leq \frac{1}{2 \eta \phi_{p}\left(\rho_{2}+1\right)} \phi_{p}(|u|+|v|), \quad \forall t \in J,|u|+|v| \geq R
\end{aligned}
$$

and we write

$$
M=\max _{\forall t \in J,|u|+|v| \leq R} F(t, u, v)
$$


Theorem 3.2 Assume that $\left(\mathrm{H}_{1}\right)-\left(\mathrm{H}_{2}\right)$ and $\left(\mathrm{H}_{4}\right)$ hold. Then we have the following conclusions:

(i) Problem (2.5) has (at least) one positive solution $x \in K$ such that

$$
\delta r \leq x(t) \leq \max \left\{2 \eta M, \phi_{p}\left(\frac{R}{\phi_{q}(\delta)\left(\rho_{1}+1\right)}\right)\right\}, \quad t \in J .
$$

(ii) Problem (1.5) has (at least) one positive solution y such that (3.4) holds. We further have

$$
\sigma \delta \phi_{q}(r) \leq y(t) \leq \max \left\{\rho_{2} \phi_{q}(2 \eta M), \frac{\rho_{2} R}{\phi_{q}(\delta)\left(\rho_{1}+1\right)}\right\}, \quad t \in J
$$

Proof Let $x \in K$ with $\|x\|=r$. Then $0 \leq \phi_{q}(x(t)) \leq \phi_{q}(r), t \in J$, and

$$
0 \leq \int_{0}^{1} H_{1}(s, \tau) \phi_{q}(x(\tau)) d \tau \leq \rho_{2} \phi_{q}(r)
$$

And hence for $x \in K$ with $\|x\|=r$, we have

$$
0 \leq\left|\int_{0}^{1} H_{1}(s, \tau) \phi_{q}(x(\tau)) d \tau\right|+\left|-\phi_{q}(x(t))\right| \leq\left(\rho_{2}+1\right) \phi_{q}(\delta r),
$$

and it follows from condition (3.10) that

$$
\begin{aligned}
\|T x\| & =\max _{t \in J} \int_{0}^{1} H_{2}(t, s) F\left(s, \int_{0}^{1} H_{1}(s, \tau) \phi_{q}(x(\tau)) d \tau,-\phi_{q}(x(s))\right) d s \\
& \geq \rho_{1} \beta \frac{1}{\rho_{1} \beta \delta \phi_{p}\left(\rho_{1}+1\right)} \phi_{p}\left[\left(\rho_{1}+1\right) \phi_{q}(\delta r)\right]=r,
\end{aligned}
$$

that is, $x \in \partial \Omega_{1}$ implies that

$$
\|T x\| \geq\|x\| .
$$

From (3.11) and (3.12), we have

$$
F(t, u, v) \leq M+\frac{1}{2 \eta \phi_{p}\left(\rho_{2}+1\right)} \phi_{p}(|u|+|v|), \quad(t, u, v) \in J \times[0, \infty) \times(-\infty, 0] .
$$

Further, let

$$
R_{2}>\max \left\{2 \eta M, \phi_{p}\left(\frac{R}{\phi_{q}(\delta)\left(\rho_{1}+1\right)}\right)\right\}
$$

and

$$
\Omega_{3}=\left\{x \in K:\|x\|<R_{2}\right\} .
$$

Noticing that for $x \in \partial \Omega_{3}$ we have

$$
R<\left(\rho_{1}+1\right) \phi_{q}\left(\delta R_{2}\right) \leq\left|\int_{0}^{1} H_{1}(s, \tau) \phi_{q}(x(\tau)) d \tau\right|+\left|-\phi_{q}(x(t))\right| .
$$


Thus, for $x \in \partial \Omega_{3}$, it follows from (3.17) that

$$
\begin{aligned}
\|T x\| & =\max _{t \in J} \int_{0}^{1} H_{2}(t, s) F\left(s, \int_{0}^{1} H_{1}(s, \tau) \phi_{q}(x(\tau)) d \tau,-\phi_{q}(x(s))\right) d s \\
& \leq \eta \int_{0}^{1}\left(M+\frac{1}{2 \eta \phi_{p}\left(\rho_{2}+1\right)} \phi_{p}\left(\left(\rho_{2}+1\right) \phi_{q}\left(R_{2}\right)\right)\right) d s \\
& =\eta M+\frac{1}{2} R_{2} \\
& <R_{2},
\end{aligned}
$$

that is, $x \in \partial \Omega_{3}$ implies

$$
\|T x\|<\|x\|
$$

It now follows from Lemma 2.6 that problem (2.5) has (at least) one positive solution $x \in \bar{\Omega}_{3} \backslash \Omega_{1}$ satisfying (3.13).

It follows from (2.2) that problem (1.5) has (at least) one positive solution $y$. Similar to the proof of (3.5), one can show that $y$ satisfies (3.14).

Theorem 3.3 Assume that $\left(\mathrm{H}_{1}\right)-\left(\mathrm{H}_{2}\right),(3.2)$ of $\left(\mathrm{H}_{3}\right)$ and (3.10) of $\left(\mathrm{H}_{4}\right)$ hold. In addition, let $f$ satisfies the following condition:

$\left(\mathrm{H}_{5}\right)$ Let $l, \zeta_{i}(i=1,2)$ and L satisfy

$$
0<l<\delta \zeta_{1}<\zeta_{1}<\delta \zeta_{2}<\zeta_{2}<L<\infty
$$

If

$$
\max _{\forall t \in J, \phi_{q}\left(\delta \zeta_{i}\right) \leq|u|+|v| \leq\left(\rho_{2}+1\right) \phi_{q}\left(\zeta_{i}\right)} F(t, u, v)<\frac{1}{\eta} \zeta_{i}, \quad i=1,2,
$$

then we have the following conclusions:

(i) Problem (2.5) has (at least) two positive solutions $x_{1}, x_{2} \in K$ such that

$$
\delta l \leq x_{1}(t) \leq \zeta_{1}<\delta \zeta_{2} \leq x_{2}(t) \leq L, \quad t \in J
$$

(ii) Problem (1.5) has (at least) two positive solutions $y_{1}, y_{2}$ such that, for $i=1,2$,

$$
\left\{\begin{array}{l}
y_{i}(t)=\int_{0}^{1} H_{1}(t, s) \phi_{q}\left(x_{i}(s)\right) d s, \quad t \in J \\
\left\|y_{i}\right\| \leq \rho_{2} \phi_{q}\left(\left\|x_{i}\right\|\right) \\
y_{i}(t) \geq \sigma \phi_{q}\left(\left\|x_{i}\right\|\right), \quad t \in J
\end{array}\right.
$$

We further have

$$
\left\{\begin{array}{l}
y_{1}(t)>\sigma \phi_{q}(l), \quad t \in J \\
\left\|y_{2}\right\| \leq \rho_{2} \phi_{q}(L)
\end{array}\right.
$$


Proof If (3.10) of $\left(\mathrm{H}_{4}\right)$ holds, similar to the proof of (3.16), we can prove that

$$
\|T x\| \geq\|x\|, \quad x \in K,\|x\|=l .
$$

If (3.2) of $\left(\mathrm{H}_{3}\right)$ holds, similar to the proof of (3.9), we have

$$
\|T x\| \geq\|x\|, \quad x \in K,\|x\|=L .
$$

Finally, we show that

$$
\|T x\|<\|x\|, \quad x \in K,\|x\|=\zeta_{i}, i=1,2 .
$$

In fact, for $x \in K$ with $\|x\|=\zeta_{i}(i=1,2)$ then we have

$$
x(t) \leq\|x\|=\zeta_{i}, \quad i=1,2,
$$

and

$$
\rho_{1} \phi_{q}\left(\delta \zeta_{i}\right) \leq \int_{0}^{1} H_{1}(s, \tau) \phi_{q}(x(\tau)) d \tau \leq \rho_{2} \phi_{q}\left(\zeta_{i}\right), \quad i=1,2 .
$$

Therefore,

$$
\begin{aligned}
& \phi_{q}\left(\delta \zeta_{i}\right)<\left(\rho_{1}+1\right) \phi_{q}\left(\delta \zeta_{i}\right) \leq\left|\int_{0}^{1} H_{1}(s, \tau) \phi_{q}(x(\tau)) d \tau\right|+\left|-\phi_{q}(x(s))\right| \leq\left(\rho_{2}+1\right) \phi_{q}\left(\zeta_{i}\right), \\
& \quad i=1,2
\end{aligned}
$$

and hence it follows from $\left(\mathrm{H}_{5}\right)$ that

$$
\begin{aligned}
\|T x\| & =\max _{t \in J} \int_{0}^{1} H_{2}(t, s) F\left(s, \int_{0}^{1} H_{1}(s, \tau) \phi_{q}(x(\tau)) d \tau,-\phi_{q}(x(s))\right) d s \\
& <\eta \frac{1}{\eta} \zeta_{i}=\zeta_{i}, \quad i=1,2,
\end{aligned}
$$

which shows that (3.25) holds.

Applying Lemma 2.6 to (3.23), (3.24) and (3.25) shows that problem (2.5) has (at least) two positive solutions $x_{1}, x_{2}$ with $x_{1} \in K_{l, \zeta_{1}}=\left\{x \in K, l \leq\|x\| \leq \zeta_{1}\right\}, x_{2} \in K_{\zeta_{2}, L}=\left\{x \in K, \zeta_{2} \leq\right.$ $\|x\| \leq L\}$. Hence, since for $x_{i} \in K$ we have $x_{i}(t) \geq \delta\left\|x_{i}\right\|, t \in J, i=1,2$, it follows that (3.20) holds.

Similar to the proof of (3.4) and (3.5), one can show that (3.21) and (3.22) hold.

In Theorem 3.4 we assume the following condition on $F(t, u, v)$ :

$\left(\mathrm{H}_{6}\right)$ There exist two constants $r, R$ with $0<r<\delta R$ such that

$$
\begin{aligned}
& F(t, u, v) \leq \frac{1}{\eta} r, \quad \forall(t, u, v) \in J \times\left[\rho_{1} \phi_{q}(\delta r), \rho_{2} \phi_{q}(r)\right] \times\left[-\phi_{q}(r),-\phi_{q}(\delta r)\right] \\
& F(t, u, v) \geq \frac{1}{\rho \beta} R, \quad \forall(t, u, v) \in J \times\left[\rho_{1} \phi_{q}(\delta R), \rho_{2} \phi_{q}(R)\right] \times\left[-\phi_{q}(R),-\phi_{q}(\delta R)\right] .
\end{aligned}
$$


Theorem 3.4 Assume that $\left(\mathrm{H}_{1}\right)-\left(\mathrm{H}_{2}\right)$ and $\left(\mathrm{H}_{6}\right)$ hold. Then we have the following conclusions:

(i) Problem (2.5) has (at least) one positive solution $x \in K$ such that

$$
\delta r \leq x(t) \leq R, \quad t \in J
$$

(ii) Problem (1.5) has (at least) one positive solution y such that (3.4) holds. We further have

$$
\sigma \phi_{q}(r) \leq y(t) \leq \rho_{2} \phi_{q}(R), \quad t \in J
$$

Proof For $x \in K$ with $\|x\|=r$, we have $x(s) \in[\delta r, r]$, and

$$
\begin{aligned}
& \int_{0}^{1} H_{1}(s, \tau) \phi_{q}(x(\tau)) d \tau \in\left[\rho_{1} \phi_{q}(\delta r), \rho_{2} \phi_{q}(r)\right], \\
& -\phi_{q}(x(s)) \in\left[-\phi_{q}(r),-\phi_{q}(\delta r)\right], \quad \forall s \in J .
\end{aligned}
$$

Then, for $t \in J$, we have

$$
\begin{aligned}
(T x)(t) & =\int_{0}^{1} H_{2}(t, s) F\left(s, \int_{0}^{1} H_{1}(s, \tau) \phi_{q}(x(\tau)) d \tau,-\phi_{q}(x(s))\right) d s \\
& \leq \int_{0}^{1} \gamma_{2} G_{2}(s, s) d s \cdot \frac{1}{\eta} r \\
& =r
\end{aligned}
$$

i.e. $x \in \partial \Omega_{1}$ implies

$$
\|T x\| \leq\|x\| .
$$

On the other hand, for $x \in K$ with $\|x\|=R$, we have $x \in[\delta R, R]$, and $\phi_{q}(x) \in\left[-\phi_{q}(R)\right.$, $\left.-\phi_{q}(\delta R)\right], \int_{0}^{1} H_{1}(s, \tau) \phi_{q}(x(\tau)) d \tau \in\left[\rho_{1} \phi_{q}(\delta R), \rho_{2} \phi_{q}(R)\right], \forall s \in J$. Then, for $t \in J$, we have

$$
\begin{aligned}
(T x)(t) & =\int_{0}^{1} H_{2}(t, s) F\left(s, \int_{0}^{1} H_{1}(s, \tau) \phi_{q}(x(\tau)) d \tau,-\phi_{q}(x(s))\right) d s \\
& \geq \int_{0}^{1} \rho G_{2}(s, s) d s \cdot \frac{6}{\rho} R \\
& =R,
\end{aligned}
$$

i.e. $x \in \partial \Omega_{2}$ implies

$$
\|T x\| \geq\|x\| .
$$

Applying Lemma 2.6 to (3.28) and (3.29) shows that $T$ has a fixed point $x \in K \cap\left(\bar{\Omega}_{2} \backslash \Omega_{1}\right)$ with (3.26). It is observed from (2.2) that problem (1.5) has one solution $y$ such that

$$
y(t)=\int_{0}^{1} H_{1}(t, s) \phi_{q}(x(s)) d s, \quad \text { and } \quad y(t) \in\left[\sigma \phi_{q}(r), \rho_{2} \phi_{q}(R)\right], \quad t \in J .
$$


We remark that the condition $\left(\mathrm{H}_{6}\right)$ in Theorem 3.4 can be replaced by the following condition:

$\left(\mathrm{H}_{7}\right)$ There exist two constants $r, R$ with $0<r<\delta R$ such that

$$
\begin{aligned}
& F(t, u, v) \geq \frac{1}{\rho \beta} r, \quad \forall(t, u, v) \in J \times\left[\rho_{1} \phi_{q}(\delta r), \rho_{2} \phi_{q}(r)\right] \times\left[-\phi_{q}(r), \phi_{q}(\delta r)\right] \\
& F(t, u, v) \leq \frac{1}{\eta} R, \quad \forall(t, u, v) \in J \times\left[\rho_{1} \phi_{q}(\delta R), \rho_{2} \phi_{q}(R)\right] \times\left[-\phi_{q}(R), \phi_{q}(\delta R)\right] .
\end{aligned}
$$

Corollary 3.1 If the condition $\left(\mathrm{H}_{6}\right)$ in Theorem 3.4 is replaced by $\left(\mathrm{H}_{7}\right)$, respectively, then the conclusions of Theorem 3.4 also hold.

\section{Further results on twin solutions}

In the previous section, we have obtained some results on the existence of at least two positive solutions for problem (1.5). In this section, we will further discuss the existence of two positive solutions for problem (1.5) in the more general case.

For $x \in K$, we define the nonnegative increasing continuous functionals $\theta, \theta_{1}$ and $\theta_{2}$ by

$$
\begin{aligned}
& \theta(x)=\min _{t \in J} x(t), \\
& \theta_{1}(x)=\max _{t \in\left[0, \frac{1}{2}\right]} x(t),
\end{aligned}
$$

and

$$
\theta_{2}(x)=\max _{t \in J} x(t)
$$

It is easy to see that, for each $x \in K$,

$$
\theta(x) \leq \theta_{1}(x) \leq \theta_{2}(x)
$$

In addition, for each $x \in K, \theta(x)=x(1) \geq \delta\|x\|$, which implies that

$$
\|x\| \leq \frac{1}{\delta} \theta(x) \quad \text { for all } x \in K .
$$

Finally, we also note that

$$
\theta_{1}(\lambda x)=\lambda \theta_{1}(x) \quad \text { for } 0 \leq \lambda \leq 1 \text { and } x \in \partial K\left(\theta_{1}, r\right)
$$

where $r>0$.

For notational convenience, we denote

$$
N=\rho \beta, \quad N_{1}=\eta .
$$

We now present the results in this section. 
Theorem 4.1 Suppose that there exist constants $0<a<b<c$ such that

$$
0<a<\frac{N}{N_{1}} b<\frac{\delta N}{N_{1}} c .
$$

Assume F satisfies the following conditions:

(C $\left.C_{1}\right) F(t, u, v)>\frac{c}{N}$ for $(t, u, v) \in J \times\left[\rho_{1} \phi_{q}(c), \rho_{2} \phi_{q}\left(\frac{c}{\delta}\right)\right] \times\left[-\phi_{q}\left(\frac{c}{\delta}\right),-\phi_{q}(c)\right]$;

$\left(C_{2}\right) F(t, u, v)<\frac{b}{N_{1}}$ for $(t, u, v) \in J \times\left[0, \rho_{2} \phi_{q}\left(\frac{b}{\delta}\right)\right] \times\left[-\phi_{q}\left(\frac{b}{\delta}\right), 0\right]$;

$\left(C_{3}\right) F(t, u, v)>\frac{a}{N} f o r(t, u, v) \in J \times\left[\rho_{1} \phi_{q}(\delta a), \rho_{2} \phi_{q}(a)\right] \times\left[-\phi_{q}(a),-\phi_{q}(\delta a)\right]$.

Then we have the following conclusions:

(i) Problem (2.5) has at least two positive solutions $x_{1}$ and $x_{2}$ such that

$$
a<\theta_{2}\left(x_{1}\right) \text {, with } \theta_{1}\left(x_{1}\right)<b \text {, and } b<\theta_{1}\left(x_{2}\right) \text { with } \theta\left(x_{2}\right)<c \text {. }
$$

(ii) Problem (1.5) has (at least) two positive solutions $y_{1}$ and $y_{2}$ such that for $i=1,2$

$$
\left\{\begin{array}{l}
y_{i}(t)=\int_{0}^{1} H_{1}(t, s) \phi_{q}\left(x_{i}(s)\right) d s, \quad t \in J \\
\left\|y_{i}\right\| \leq \rho_{2} \phi_{q}\left(\left\|x_{i}\right\|\right) \\
y_{i}(t) \geq \sigma \phi_{q}\left(\left\|x_{i}\right\|\right), \quad t \in J
\end{array}\right.
$$

We further have

$$
\left\{\begin{array}{l}
\left\|y_{1}\right\|>\sigma \phi_{q}(a) ; \\
y_{2}(t)<\rho_{2} \phi_{q}\left(\|x\|_{2}\right), \quad t \in J .
\end{array}\right.
$$

Proof By the definition of operator $T$ and its properties, it suffices to show that the conditions of Lemma 2.7 hold with respect to $T$.

We first show that if $x \in \partial K(\theta, c)$, then $\theta(T x)>c$.

In fact, if $x \in \partial K(\theta, c)$, then $\theta(x)=\min _{t \in J} x(t)=x(1)=c$. Since $x \in K$, one can get $x(t) \geq c$ for $t \in J$. Noticing (4.2), we have

$$
c \leq x(t) \leq \frac{1}{\delta} c, \quad t \in J,
$$

which shows that

$$
\rho_{1} \phi_{q}(c) \leq \int_{0}^{1} H_{1}(t, s) \phi_{q}(x(s)) d s \leq \rho_{2} \phi_{q}\left(\frac{1}{\delta} c\right), \quad-\phi_{q}\left(\frac{1}{\delta} c\right) \leq-\phi_{q}(x(s)) \leq-\phi_{q}(c) .
$$

As a consequence of $\left(C_{1}\right)$

$$
F(t, u, v)>\frac{c}{N} \quad \text { for } t \in J .
$$

Also, $T x \in K$, and hence we get

$$
\begin{aligned}
\theta(T x) & =\min _{t \in J}(T x)(t)=(T x)(0) \\
& =\min _{t \in J} \int_{0}^{1} H_{2}(t, s) F\left(s, \int_{0}^{1} H_{1}(s, \tau) \phi_{q}(x(\tau)),-\phi_{q}(x(s))\right) d s
\end{aligned}
$$




$$
\begin{aligned}
& >\rho \beta \frac{c}{N} \\
& =c .
\end{aligned}
$$

Next, we verify that $\theta_{1}(T x)<b$ for $x \in \partial K\left(\theta_{1}, b\right)$.

So, let us choose $x \in \partial K\left(\theta_{1}, b\right)$, then

$$
\theta_{1}(x)=\max _{t \in\left[0, \frac{1}{2}\right]} x(t)=b .
$$

This implies that $0 \leq x(t) \leq b$ for $t \in\left[0, \frac{1}{2}\right]$, and, since $x \in K$, we also have

$$
x(t) \leq\|x\| \leq \frac{1}{\delta} \theta(x) \leq \frac{1}{\delta} \theta_{1}(x)=\frac{1}{\delta} b,
$$

which implies that

$$
0 \leq x(t) \leq \frac{1}{\delta} b, \quad t \in J
$$

and then

$$
0 \leq \int_{0}^{1} H_{1}(t, s) \phi_{q}(s) d s \leq \rho_{2} \phi_{q}\left(\frac{1}{\delta} b\right), \quad-\phi_{q}\left(\frac{1}{\delta} b\right) \leq-\phi_{q}(x(s)) \leq 0 .
$$

It follows from $\left(C_{2}\right)$ that

$$
F(t, u, v)<\frac{b}{N_{1}} \quad \text { for }(t, u, v) \in J \times\left[0, \rho_{2} \phi_{q}\left(\frac{b}{\delta}\right)\right] \times\left[-\phi_{q}\left(\frac{b}{\delta}\right), 0\right] .
$$

Noticing $T x \in K$, we obtain

$$
\begin{aligned}
(T x)(t) & =\int_{0}^{1} H_{2}(t, s) F\left(s, \int_{0}^{1} H_{1}(s, \tau) \phi_{q}(x(\tau)),-\phi_{q}(x(s))\right) d s \\
& <\eta \frac{b}{N_{1}} \\
& =b .
\end{aligned}
$$

Finally, we prove that $K\left(\theta_{2}, a\right) \neq \emptyset$ and $\theta_{2}(T x)>a$ for all $x \in \partial K\left(\theta_{2}, a\right)$. In fact, the constant $\frac{a}{3} \in K\left(\theta_{2}, a\right)$. Moreover, for $x \in \partial K\left(\theta_{2}, a\right)$, we have

$$
\theta_{2}(x)=\max _{t \in J} x(t)=a,
$$

which implies that

$$
\delta a \leq x(t) \leq a, \quad t \in J
$$

and then

$$
\rho_{1} \phi_{q}\left(\frac{1}{\delta} a\right) \leq \int_{0}^{1} H_{1}(t, s) \phi_{q}(s) d s \leq \rho_{2} \phi_{q}(a), \quad-\phi_{q}(a) \leq-\phi_{q}(x(s)) \leq-\phi_{q}\left(\frac{1}{\delta} a\right) .
$$


Using assumption $\left(\mathrm{C}_{3}\right)$, we have

$$
F(t, u, v)>\frac{a}{N} \quad \text { for }(t, u, v) \in J \times\left[\rho_{1} \phi_{q}(\delta a), \rho_{2} \phi_{q}(a)\right] \times\left[-\phi_{q}(a),-\phi_{q}(\delta a)\right] .
$$

As before $T x \in K$, and so

$$
\begin{aligned}
\theta_{2}(T x) & =\max _{t \in J}(T x)(t) \\
& =\max _{t \in J} \int_{0}^{1} H_{2}(t, s) F\left(s, \int_{0}^{1} H_{1}(s, \tau) \phi_{q}(x(\tau)),-\phi_{q}(x(s))\right) d s \\
& >\rho \eta \frac{a}{N} \\
& =a .
\end{aligned}
$$

Thus, by Lemma 2.7, there exist at least two fixed points of $T$ which are positive solutions $x_{1}$ and $x_{2}$, belonging to $\overline{K(\theta, c)}$, of problem (2.5) such that

$$
a<\theta_{2}\left(x_{1}\right), \quad \text { with } \theta_{1}\left(x_{1}\right)<b, \quad \text { and } \quad b<\theta_{1}\left(x_{2}\right) \quad \text { with } \theta\left(x_{2}\right)<c \text {. }
$$

Noticing the definition of $\theta$ and $\theta_{2}$, one can show that (4.4) and (4.5) hold.

\section{Triple positive solutions}

In this section, we shall study the existence of three positive solutions of problem (1.5). Let $\sigma, N$ and $N_{1}$ be defined in Theorem 3.1 and Theorem 4.1, respectively.

Theorem 5.1 Assume that $\left(\mathrm{H}_{1}\right)-\left(\mathrm{H}_{2}\right)$ hold. Suppose that there exist positive constants $\omega_{1}$, $\omega_{2}, \omega_{3}$ with

$$
0<\omega_{1}<\phi_{p}\left(\frac{\rho_{1}}{\rho_{2}}\right) \delta \omega_{2}<\omega_{2}<\frac{\omega_{2}}{\delta} \leq \omega_{3}
$$

such that the following hold:

$\left(\mathrm{A}_{1}\right) F(t, u, v) \leq \frac{1}{N_{1}} \omega_{3}$ for $(t, u, v) \in J \times\left[0, \rho_{2} \phi_{q}\left(\omega_{3}\right)\right] \times\left[-\phi_{q}\left(\omega_{3}\right), 0\right]$;

$\left(\mathrm{A}_{2}\right) F(t, u, v)>\frac{1}{N} \omega_{2}$ for $(t, u, v) \in J \times\left[\rho_{1} \phi_{q}\left(\omega_{2}\right), \rho_{2} \phi_{q}\left(\frac{1}{\delta} \omega_{2}\right)\right] \times\left[-\phi_{q}\left(\frac{1}{\delta} \omega_{2}\right),-\phi_{q}\left(\omega_{2}\right)\right]$;

$\left(\mathrm{A}_{3}\right) F(t, u, v)<\frac{1}{N_{1}} \omega_{1}$ for $(t, u, v) \in J \times\left[0, \rho_{2} \phi_{q}\left(\omega_{1}\right)\right] \times\left[-\phi_{q}\left(\omega_{1}\right), 0\right]$.

Then we have the following conclusions:

(i) Problem (2.5) has (at least) three positive solutions $x_{1}, x_{2}, x_{3} \in K$ such that

$$
\left\{\begin{array}{l}
\left\|x_{1}\right\|<\omega_{1} ; \\
x_{2}(t)>\omega_{2}, \quad t \in J \\
\left\|x_{3}\right\|>\omega_{1} \text { and } \min _{t \in J} x_{3}(t)<\omega_{2} .
\end{array}\right.
$$

(ii) Problem (1.5) has (at least) three positive solutions $y_{1}, y_{2}, y_{3}$ such that, for $i=1,2,3$,

$$
\left\{\begin{array}{l}
y_{i}(t)=\int_{0}^{1} H_{1}(t, s) \phi_{q}\left(x_{i}(s)\right) d s, \quad t \in J \\
\left\|y_{i}\right\| \leq \rho_{2} \phi_{q}\left(\left\|x_{i}\right\|\right) \\
y_{i}(t) \geq \rho_{1} \phi_{q}\left(\delta\left\|x_{i}\right\|\right), \quad t \in J
\end{array}\right.
$$


We further have

$$
\left\{\begin{array}{l}
\left\|y_{1}\right\|<\rho_{2} \phi_{q}\left(\omega_{1}\right) ; \\
y_{2}(t)>\rho_{1} \phi_{q}\left(\omega_{2}\right), \quad t \in J ; \\
\left\|y_{3}\right\|>\sigma \phi_{q}\left(\omega_{1}\right) .
\end{array}\right.
$$

Proof Let $x \in \bar{K}_{\omega_{3}}$, so $0 \leq \phi_{q}(x(s)) \leq \phi_{q}\left(\omega_{3}\right), s \in J$, and $0 \leq \int_{0}^{1} H_{1}(s, \tau) \phi_{q}(x(\tau)) d \tau \leq$ $\rho_{2} \phi_{q}\left(\omega_{3}\right)$. By $\left(\mathrm{A}_{1}\right)$, we see that

$$
\begin{aligned}
\|T x\| & =\max _{t \in J} \int_{0}^{1} H_{2}(t, s) F\left(s, \int_{0}^{1} H_{1}(s, \tau) \phi_{q}(x(\tau)) d \tau,-\phi_{q}(x(s))\right) d s \\
& \leq \eta \cdot \frac{1}{N_{1}} \omega_{3}=\omega_{3} .
\end{aligned}
$$

This proves that $T: \bar{K}\left(\omega_{3}\right) \rightarrow \bar{K}\left(\omega_{3}\right)$.

For convenience, we denote $\omega_{4}=\frac{\omega_{2}}{\delta}$. Let $\psi: K \rightarrow[0,+\infty)$ be defined by

$$
\psi(x)=\min _{t \in J} x(t)
$$

Clearly, $\psi$ is a nonnegative continuous concave functional on $K$ and $\psi(x) \leq\|x\|$ for all $x \in K$.

Now we need to show that condition (i) of Lemma 2.8 is satisfied. It is obvious that

$$
x(t)=\frac{1}{2}\left(\omega_{2}+\omega_{4}\right) \in\left\{x \in K\left(\psi, \omega_{2}, \omega_{4}\right): \psi(x)>\omega_{2}\right\}
$$

Then

$$
\left\{x \in K\left(\psi, \omega_{2}, \omega_{4}\right): \psi(x)>\omega_{2}\right\} \neq \emptyset .
$$

Next, let $x \in K\left(\psi, \omega_{2}, \omega_{4}\right)$. Then for $s \in J$, we have $\omega_{2} \leq x(s) \leq \omega_{4}$, and

$$
\int_{0}^{1} H_{1}(s, \tau) \phi_{q}(x(\tau)) d \tau \in\left[\rho_{1} \phi_{q}\left(\omega_{2}\right), \rho_{2} \phi_{q}\left(\omega_{4}\right)\right]
$$

Using condition $\left(\mathrm{A}_{2}\right)$, it follows that

$$
\begin{aligned}
\psi(T x) & =\min _{t \in J} \int_{0}^{1} H_{2}(t, s) F\left(s, \int_{0}^{1} H_{1}(s, \tau) \phi_{q}(x(\tau)) d \tau,-\phi_{q}(x(s))\right) d s \\
& \geq \rho \int_{0}^{1} G_{2}(s, s) F\left(s, \int_{0}^{1} H_{1}(s, \tau) \phi_{q}(x(\tau)) d \tau,-\phi_{q}(x(s))\right) d s \\
& >\rho \beta \cdot \frac{1}{N} \omega_{2} \\
& =\omega_{2} .
\end{aligned}
$$

Therefore, we have shown that $\psi(T x)>\omega_{2}$ for all $x \in K\left(\psi, \omega_{2}, \omega_{4}\right)$. 
Next, we shall show that condition (ii) of Lemma 2.8 holds. Take $x \in \bar{K}\left(\omega_{1}\right)$ with $\|x\|<\omega_{1}$. Then for $s \in J$, we have $x(s) \in\left[0, \omega_{1}\right]$ and

$$
\int_{0}^{1} H_{1}(s, \tau) \phi_{q}(x(\tau)) d \tau \in\left[0, \rho_{2} \phi_{q}\left(\omega_{1}\right)\right]
$$

By condition $\left(\mathrm{A}_{3}\right)$, we have

$$
\|T x\|=\max _{t \in J} \int_{0}^{1} H_{2}(t, s) F\left(s, \int_{0}^{1} H_{1}(s, \tau) \phi_{q}(x(\tau)) d \tau,-\phi_{q}(x(s))\right) d s<\eta \cdot \frac{1}{N_{1}} \omega_{1}=\omega_{1} .
$$

Hence, we have proved that $\|T x\|<\omega_{1}$ for all $x \in \bar{K}\left(\omega_{1}\right)$.

Finally, we need to prove that condition (iii) of Lemma 2.8 is satisfied. Let $x \in$ $K\left(\psi, \omega_{2}, \omega_{3}\right)$ with $\|T x\|>\omega_{4}$. Then it follows from (2.16) that

$$
\begin{aligned}
\psi(T x) & =\min _{t \in J} \int_{0}^{1} H_{2}(t, s) F\left(s, \int_{0}^{1} H_{1}(s, \tau) \phi_{q}(x(\tau)) d \tau,-\phi_{q}(x(s))\right) d s \\
& \geq \delta\|T x\| \\
& >\delta \omega_{4}=\omega_{2} .
\end{aligned}
$$

So, we have proved that $\psi(T x)>\omega_{2}$ for all $x \in K\left(\psi, \omega_{2}, \omega_{3}\right)$ with $\|T x\|>\omega_{4}$.

It now follows from Lemma 2.8 that problem (2.5) has (at least) three positive solutions $x_{1}, x_{2}, x_{3} \in \bar{K}\left(\omega_{3}\right)$ satisfying (5.1).

It is observed from (2.2) that problem (1.5) has (at least) three positive solutions $y_{1}, y_{2}$, $y_{3}$ such that, for $i=1,2,3$,

$$
y_{i}(t)=\int_{0}^{1} H_{1}(t, s) \phi_{q}\left(x_{i}(s)\right) d s, \quad t \in J \text { and }\left\|y_{i}\right\| \leq \rho_{2} \phi_{q}\left(\left\|x_{i}\right\|\right) .
$$

Moreover, since $x_{i} \in K$, we get for $t \in J$,

$$
\begin{aligned}
y_{i}(t) & =\int_{0}^{1} H_{1}(t, s) \phi_{q}\left(x_{i}(s)\right) d s \\
& \geq \rho_{1} \int_{0}^{1} \phi_{q}\left(x_{i}(s)\right) d s \\
& \geq \sigma \phi_{q}\left(\left\|x_{i}\right\|\right) .
\end{aligned}
$$

Then we get (5.2).

Further, since $x_{2}(t)>\omega_{2}$ for $t \in J$, we have, for $t \in J$,

$$
\begin{aligned}
y_{2}(t) & =\int_{0}^{1} H_{1}(t, s) \phi_{q}\left(x_{2}(s)\right) d s \\
& \geq \rho_{1} \int_{0}^{1} \phi_{q}\left(x_{2}(s)\right) d s \\
& \geq \rho_{1} \phi_{q}\left(\omega_{2}\right) .
\end{aligned}
$$

Hence, we get (5.3). 
Remark 5.1 Leggett-Williams' fixed point theorem is a valid tool to show the existence of multiple positive solutions for various boundary value problems where the nonlinear terms $F$ is of the form $F(t, y)$; see for example [25-27]. If $F$ is of the form $F\left(t, y, y^{\prime \prime}\right)$, then it is very difficult to obtain the existence result of three positive solutions; see [20, 21] to name a few. In [28], using the five functionals fixed point theorem (which is a generalization of the Leggett-Williams' fixed point theorem), Avery et al. showed the existence of at least three positive solutions in the case that $F$ is the form of $F\left(y,-y^{\prime \prime}\right)$.

\section{Example}

To illustrate how our main results can be used in practice, we present an example.

Let $a=1, b=\frac{1}{2}$ and $g(t)=h(t)=\frac{1}{2}$. Consider the following fourth order $p$-Laplacian elasticity problem:

$$
\left\{\begin{array}{l}
\left(\phi_{p}\left(y^{\prime \prime}(t)\right)\right)^{\prime \prime}=F\left(t, y(t), y^{\prime \prime}(t)\right), \quad 0<t<1, \\
a y(0)-b y^{\prime}(0)=\int_{0}^{1} g(s) y(s) d s, \\
a y(1)+b y^{\prime}(1)=\int_{0}^{1} g(s) y(s) d s, \\
\phi_{p}\left(y^{\prime \prime}(0)\right)=\phi_{p}\left(y^{\prime \prime}(1)\right)=\int_{0}^{1} h(s) \phi_{p}\left(y^{\prime \prime}(s)\right) d s
\end{array}\right.
$$

and the nonlinear term $F$ given by

$$
F(t, u, v)=\left\{\begin{array}{l}
\frac{1}{2}\left(t^{2}+1\right) \frac{1}{5 N_{1}} \omega_{1}=: a_{1}(t), \quad t \in J,(u, v) \in D_{1}, \\
\frac{1}{5}\left(\frac{\omega_{3}}{N_{1}}+\frac{\omega_{2}}{N}\right)=: a_{2}, \quad t \in J,(u, v) \in D_{2} \cup D_{3} \cup D_{4}, \\
a_{1}(t) \cdot \frac{\rho_{1} \phi_{q}\left(\omega_{2}\right)-u}{\rho_{1} \phi_{q}\left(\omega_{2}\right)-\rho_{2} \phi_{q}\left(\omega_{1}\right)}+a_{2} \cdot \frac{u-\rho_{2} \phi_{q}\left(\omega_{1}\right)}{\rho_{1} \phi_{q}\left(\omega_{2}\right)-\rho_{2} \phi_{q}\left(\omega_{1}\right)}=: a_{5}(t, u), \quad t \in J,(u, v) \in D_{5}, \\
a_{1}(t) \cdot \frac{\phi\left(\omega_{2}\right)+v}{\phi_{q}\left(\omega_{2}\right)-\phi_{q}\left(\omega_{1}\right)}+a_{2} \cdot \frac{\phi_{q}\left(\omega_{1}\right)+v}{\phi_{q}\left(\omega_{1}\right)-\phi_{q}\left(\omega_{2}\right)}=: a_{6}(t, v), \quad t \in J,(u, v) \in D_{6}, \\
a_{7}(t, u, v), \quad t \in J,(u, v) \in D_{7},
\end{array}\right.
$$

where

$$
\begin{aligned}
a_{7}(t, u, v):= & \frac{a_{2}}{2} \cdot\left[\frac{\phi_{q}\left(\omega_{1}\right)+v}{\phi_{q}\left(\omega_{1}\right)-\phi_{q}\left(\omega_{2}\right)}+\frac{u-\rho_{2} \phi_{q}\left(\omega_{1}\right)}{\rho_{1} \phi_{q}\left(\omega_{2}\right)-\rho_{2} \phi_{q}\left(\omega_{1}\right)}\right] \\
& +\frac{a_{5}(t, u)}{2} \cdot \frac{\phi_{q}\left(\omega_{2}\right)+v}{\phi_{q}\left(\omega_{2}\right)-\phi_{q}\left(\omega_{1}\right)}+\frac{a_{6}(t, v)}{2} \cdot \frac{\rho_{1} \phi_{q}\left(\omega_{2}\right)-u}{\rho_{1} \phi_{q}\left(\omega_{2}\right)-\rho_{2} \phi_{q}\left(\omega_{1}\right)},
\end{aligned}
$$

and the sets $D_{i}(i=1,2, \ldots, 7)$ are defined by

$$
\begin{aligned}
& D_{1}=\left\{(u, v):(u, v) \in\left[0, \rho_{2} \phi_{q}\left(\omega_{1}\right)\right] \times\left[-\phi_{q}\left(\omega_{1}\right), 0\right]\right\}, \\
& D_{2}=\left\{(u, v):(u, v) \in\left[\rho_{1} \phi_{q}\left(\omega_{2}\right),+\infty\right) \times\left(-\infty,-\phi_{q}\left(\omega_{2}\right)\right]\right\}, \\
& D_{3}=\left\{(u, v):(u, v) \in\left[0, \rho_{1} \phi_{q}\left(\omega_{2}\right)\right] \times\left(-\infty,-\phi_{q}\left(\omega_{2}\right)\right]\right\}, \\
& D_{4}=\left\{(u, v):(u, v) \in\left[\rho_{1} \phi_{q}\left(\omega_{2}\right),+\infty\right) \times\left[-\phi_{q}\left(\omega_{2}\right), 0\right]\right\}, \\
& D_{5}=\left\{(u, v):(u, v) \in\left[\rho_{2} \phi_{q}\left(\omega_{1}\right), \rho_{1} \phi_{q}\left(\omega_{2}\right)\right] \times\left[-\phi_{q}\left(\omega_{1}\right), 0\right]\right\}, \\
& D_{6}=\left\{(u, v):(u, v) \in\left[0, \rho_{2} \phi_{q}\left(\omega_{1}\right)\right] \times\left[-\phi_{q}\left(\omega_{2}\right),-\phi_{q}\left(\omega_{1}\right)\right]\right\}, \\
& D_{7}=\left\{(u, v):(u, v) \in\left[\rho_{2} \phi_{q}\left(\omega_{1}\right), \rho_{1} \phi_{q}\left(\omega_{2}\right)\right] \times\left[-\phi_{q}\left(\omega_{2}\right),-\phi_{q}\left(\omega_{1}\right)\right]\right\},
\end{aligned}
$$

and $N=\rho \beta, N_{1}=\eta$. For details, see Figure 1 . 


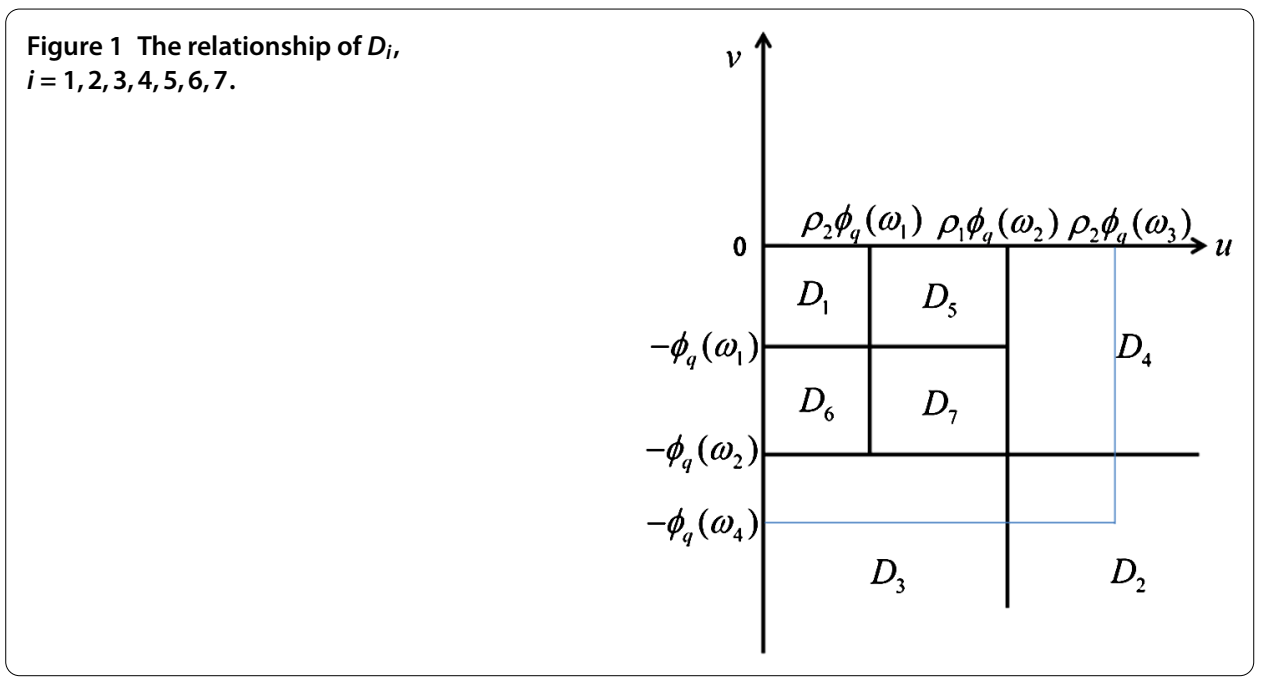

By calculation we obtain

$$
\begin{aligned}
& \mu=\int_{0}^{1} g(s) d s=\frac{1}{2}, \quad v=\int_{0}^{1} h(s) d s=\frac{1}{2}, \\
& \gamma_{1}=\frac{1}{a-\mu}=2, \quad \gamma_{2}=\frac{1}{1-v}=2, \\
& \rho=\frac{\int_{0}^{1} G_{2}(s, s) h(s) d s}{1-v}=\frac{1}{6}, \quad \eta=\frac{1}{4} \gamma_{2}=\frac{1}{2}, \\
& \rho_{1}=\frac{b^{2} \gamma_{1}}{a+2 b}=\frac{1}{4}, \quad \rho_{2}=\frac{(a+b)^{2} \gamma_{1}}{a+2 b}=\frac{9}{4} .
\end{aligned}
$$

Therefore, it follows from the definitions $F, g$ and, $h$ that $\left(\mathrm{H}_{1}\right)-\left(\mathrm{H}_{2}\right)$ hold.

Choosing $\omega_{1}=\phi_{p}\left(\frac{\delta \rho_{1}}{2 \rho_{2}}\right) \omega_{2}, \omega_{3}=\frac{5 N_{1}}{N} \omega_{2}$, then $\omega_{i}(i=1,2,3)$ satisfy

$$
0<\omega_{1}<\phi_{p}\left(\frac{\rho_{1}}{\rho_{2}}\right) \omega_{2}<\omega_{2}<\frac{\omega_{2}}{\delta} \leq \omega_{3}
$$

Then, for $t \in J,(u, v) \in D_{1}$, we have

$$
F(t, u, v)=a_{1}(t) \leq \frac{\omega_{1}}{5 N_{1}}<\frac{\omega_{3}}{5 N_{1}}<\frac{\omega_{3}}{N_{1}}
$$

For $t \in J,(u, v) \in D_{2} \cup D_{3} \cup D_{4}$, we have

$$
\begin{aligned}
& F(t, u, v)=a_{2}=\frac{1}{5}\left(\frac{\omega_{3}}{N_{1}}+\frac{\omega_{2}}{N}\right)=\frac{1}{5}\left(\frac{5 \omega_{2}}{N}+\frac{\omega_{2}}{N}\right)>\frac{\omega_{2}}{N} \\
& F(t, u, v)=a_{2}=\frac{1}{5}\left(\frac{\omega_{3}}{N_{1}}+\frac{\omega_{2}}{N}\right)=\frac{1}{5}\left(\frac{\omega_{3}}{N_{1}}+\frac{\omega_{3}}{5 N_{1}}\right)=\frac{6}{25 N_{1}} \omega_{3}<\frac{1}{N_{1}} \omega_{3} .
\end{aligned}
$$

For $t \in J,(u, v) \in D_{5}$, we have

$$
F(t, u, v)=a_{5}(u) \leq a_{1}(t)+a_{2}<\frac{\omega_{3}}{5 N_{1}}+\frac{6}{25} \cdot \frac{\omega_{3}}{N_{1}}=\frac{11}{25} \cdot \frac{\omega_{3}}{N_{1}}<\frac{\omega_{3}}{N_{1}} .
$$


For $t \in J,(u, v) \in D_{6}$, we have

$$
F(t, u, v)=a_{6}(v) \leq a_{1}(t)+a_{2}<\frac{\omega_{3}}{N_{1}}
$$

For $t \in J,(u, v) \in D_{7}$, we have

$$
F(t, u, v)=a_{7}(u, v) \leq a_{1}(t)+2 a_{2}<\frac{\omega_{3}}{5 N_{1}}+2 \cdot \frac{6}{25} \frac{\omega_{3}}{N_{1}}=\frac{17}{25} \frac{\omega_{3}}{N_{1}}<\frac{\omega_{3}}{N_{1}}
$$

We shall check the conditions of Theorem 5.1. First, condition $\left(A_{3}\right)$ is obviously satisfied by (6.2). Next, from (6.3)-(6.6), it follows that

$$
F(t, u, v)<\frac{\omega_{3}}{N_{1}}, \quad t \in J,(u, v) \in\left[0, \rho_{2} \phi_{q}\left(\omega_{3}\right)\right] \times\left[-\phi_{q}\left(\omega_{3}\right), 0\right]
$$

Hence, condition $\left(\mathrm{A}_{1}\right)$ holds. Finally, by $(6.3)$, we see that

$$
\begin{aligned}
F(t, u, v)=a_{2} & >\frac{\omega_{2}}{N} \\
t \in J,(u, v) & \in\left[\rho_{1} \phi_{q}\left(\delta \omega_{2}\right), \rho_{2} \phi_{q}\left(\frac{1}{\delta} \omega_{2}\right)\right] \times\left[-\phi_{q}\left(\frac{1}{\delta} \omega_{2}\right),-\phi_{q}\left(\omega_{2}\right)\right] \subset D_{2} .
\end{aligned}
$$

Condition $\left(\mathrm{A}_{2}\right)$ is satisfied.

It follows from Theorem 5.1 that problem (6.1) has (at least) three positive solutions $y_{1}$, $y_{2}, y_{3}$ such that

$$
\left\{\begin{array}{l}
\left\|y_{1}\right\|<\rho_{2} \phi_{q}\left(\omega_{1}\right) \\
y_{2}(t)>\rho_{1} \phi_{q}\left(\omega_{2}\right), \quad t \in J \\
\left\|y_{3}\right\|>\sigma \phi_{q}\left(\omega_{1}\right)
\end{array}\right.
$$

\section{Competing interests}

The authors declare that they have no competing interests.

Authors' contributions

All results belong to $Y Z$ and $X Z$. All authors read and approved the final manuscript.

\section{Acknowledgements}

This work is sponsored by the project NSFC (11301178) and the Fundamental Research Funds for the Central Universities (2014ZZD10,2014MS58). The authors are grateful to the anonymous referees for their constructive comments and suggestions, which have greatly improved this paper.

Received: 13 August 2015 Accepted: 2 November 2015 Published online: 09 November 2015

\section{References}

1. Guo, D, Lakshmikantham, V, Liu, X: Nonlinear Integral Equations in Abstract Spaces. Kluwer Academic, Dordrecht (1996)

2. Guo, D, Lakshmikantham, V: Nonlinear Problems in Abstract Cones. Academic Press, New York (1988)

3. Deimling, K: Nonlinear Functional Analysis. Springer, Berlin (1985)

4. Zhang, X, Yang, X, Ge, W: Positive solutions of $n$ th-order impulsive boundary value problems with integral boundary conditions in Banach spaces. Nonlinear Anal. 71, 5930-5945 (2009)

5. Wong, PJY: Triple solutions of complementary Lidstone boundary value problems via fixed point theorems. Bound. Value Probl. 2014, 125 (2014)

6. Bernis, F: Compactness of the support in convex and non-convex fourth order elasticity problems. Nonlinear Anal. 6 , 1221-1243 (1982)

7. Bai, Z, Huang, B, Ge, W: The iterative solutions for some fourth-order $p$-Laplace equation boundary value problems. Appl. Math. Lett. 19, 8-14 (2006)

8. Gupta, CP: Existence and uniqueness theorems for a bending of an elastic beam equation. Appl. Anal. 26, 289-304 (1988) 
9. Agarwal, RP: Boundary Value Problems for Higher Order Differential Equations. World Scientific, Singapore (1986)

10. Graef, JR, Yang, B: Existence and nonexistence of positive solutions of fourth order nonlinear boundary value problems. Appl. Anal. 74, 201-214 (2000)

11. Yao, Q: Monotonically iterative method of nonlinear cantilever beam equations. Appl. Math. Comput. 205, 432-437 (2008)

12. Cabada, A, Cid, J, Sanchez, L: Positivity and lower and upper solutions for fourth order boundary value problems. Nonlinear Anal. 67, 1599-1612 (2007)

13. Yang, $L, C$ Chen, $H$, Yang, $X$ : The multiplicity of solutions for fourth-order equations generated from a boundary condition. Appl. Math. Lett. 24, 1599-1603 (2011)

14. Li, S, Zhang, Q: Existence and uniqueness of monotone positive solutions for an elastic beam equation with nonlinear boundary conditions. Comput. Math. Appl. 63, 1355-1360 (2012)

15. Cabada, A, Tersian, S: Multiplicity of solutions of a two point boundary value problem for a fourth-order equation. Appl. Math. Comput. 219, 5261-5267 (2013)

16. Ma, T: Positive solutions for a beam equation on a nonlinear elastic foundation. Math. Comput. Model. 39, 1195-1201 (2004)

17. Bonanno, G, Bella, B: A boundary value problem for fourth-order elastic beam equations. J. Math. Anal. Appl. 343, 1166-1176 (2008)

18. Sun, J, Wang, X: Monotone positive solutions for an elastic beam equation with nonlinear boundary conditions. Math. Probl. Eng. 2011, 609189 (2011)

19. Ma, R, Zhang, J, Fu, S: The method of lower and upper solutions for fourth-order two-point boundary value problems. J. Math. Anal. Appl. 215, 415-422 (1997)

20. $\mathrm{Li}, \mathrm{Y}$ : On the existence of positive solutions for the bending elastic beam equations. Appl. Math. Comput. 189, 821-827 (2007)

21. Zhang, X, Ge, W: Positive solutions for a class of boundary-value problems with integral boundary conditions. Comput. Math. Appl. 58, 203-215 (2009)

22. Zhang, X, Feng, M: Positive solutions for classes of multi-parameter fourth-order impulsive differential equations with one-dimensional singular $p$-Laplacian. Bound. Value Probl. 2014, 122 (2014)

23. Avery, R, Henderson, J: Two positive fixed points of nonlinear operator on ordered Banach spaces. Commun. Appl. Nonlinear Anal. 8, 27-36 (2001)

24. Leggett, R, Williams, L: Multiple positive fixed points of nonlinear operators on ordered Banach spaces. Indiana Univ. Math. J. 28, 673-688 (1979)

25. Avery, R: Existence of multiple positive solutions to a conjugate boundary value problem. Math. Sci. Res. Hot-Line 2, 1-6 (1998)

26. Sun, H, Li, W: Existence theory for positive solutions to one-dimensional $p$-Laplacian boundary value problems on time scales. J. Differ. Equ. 240, 217-248 (2007)

27. Zhou, J, Feng, M: Triple positive solutions for a second order $m$-point boundary value problem with a delayed argument. Bound. Value Probl. 2015, 1 (2015)

28. Avery, R, Davis, J, Henderson, J: Three symmetric positive solutions for Lidstone problems by a generalization of the Leggett-Williams theorem. Electron. J. Differ. Equ. 2000, 40 (2000)

\section{Submit your manuscript to a SpringerOpen ${ }^{\circ}$ journal and benefit from:}

- Convenient online submission

Rigorous peer review

- Immediate publication on acceptance

- Open access: articles freely available online

- High visibility within the field

- Retaining the copyright to your article 